GA-A22752

CONF-971065--

\title{
SYSTEM FOR TUNERLESS OPERATION OF A FOUR-ELEMENT PHASED ARRAY ANTENNA FOR FAST WAVE CURRENT DRIVE
}

\author{
by \\ R.I. PINSKER, F.W. BAITY, W.P. CARY, C.C. PETTY, R.W. CALLIS, \\ J.S. deGRASSIE, and W.C. MARTIN
}

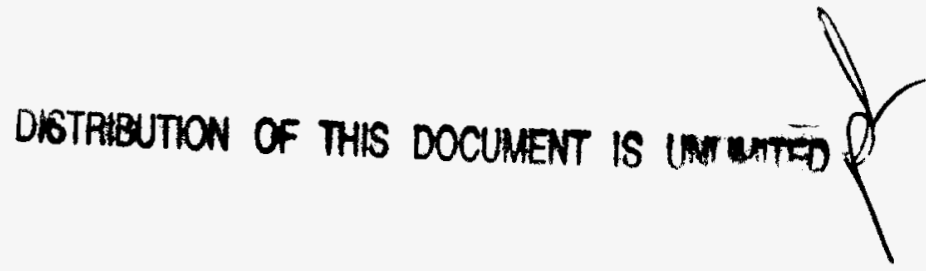

\author{
MASTER \\ RECEIVED \\ FFR 101998 \\ OSTI
}

"MIL QUALTT TISPTEUTHD 8

NOVEMBER 1997 


\section{DISCLAIMER}

This report was prepared as an account of work sponsored by an agency of the United States Government. Neither the United States Government nor any agency thereof, nor any of their employees, makes any warranty, express or implied, or assumes any legal liability or responsibility for the accuracy, completeness, or usefulness of any information, apparatus, produce, or process disclosed, or represents that its use would not infringe privately owned rights. Reference herein to any specific commercial product, process, or service by trade name, trademark, manufacturer, or otherwise, does not necessarily constitute or imply its endorsement, recommendation, or favoring by the United States Government or any agency thereof. The views and opinions of authors expressed herein do not necessarily state or reflect those of the United States Government or any agency thereof. 


\title{
SYSTEM FOR TUNERLESS OPERATION OF A FOUR-ELEMENT PHASED ARRAY ANTENNA FOR FAST WAVE CURRENT DRIVE
}

\author{
by \\ R.I. PINSKER, F.W. BAITY, † W.P. CARY, C.C. PETTY, R.W. CALLIS, \\ J.S. deGRASSIE, and W.C. MARTIN†
}

toak Ridge National Laboratory

This is a preprint of a paper to be presented at the 17th IEEE/NPSS

Symposium on Fusion Engineering, October 6-11, 1997, San Diego, California and to be published in the Proceedings.

\author{
Work supported by \\ the U.S. Department of Energy \\ under Contract No. DE-AC03-89ER51114
}




\title{
System for Tunerless Operation of a Four-Element Phased Array Antenna for Fast Wave Current Drive*
}

\author{
R.I. Pinsker, ${ }^{a}$ F.W. Baity, ${ }^{\text {b W.P. Cary, }},{ }^{\text {a C.C. Petty }},{ }^{\mathrm{a}}$ R.W. Callis, ${ }^{\mathrm{a}}$ J.S. deGrassie, ${ }^{\mathrm{a}}$ and W.C. Martin ${ }^{\mathrm{a}}$ \\ ${ }^{a}$ General Atomics, P.O. Box 85608, San Diego, California 92186-9784 \\ $\mathrm{b}_{\text {Oak Ridge National Laboratory, Oak Ridge, Tennessee 37831-8071 }}$
}

\begin{abstract}
A simple transmission line configuration for powering a four-element phased antenna array is described. This system, called the balanced feed configuration (BFC) is suitable for co- or counter- Fast Wave Current Drive (FWCD) applications. It has the property of presenting a constant matched load to the transmitter despite wide variations in the antenna load impedance without the use of variable tuning elements. This system has been implemented on a $2 \mathrm{MW} 60 \mathrm{MHz}$ FWCD antenna on the DIII-D tokamak.
\end{abstract}

\section{INTRODUCTION}

Recent work at DIII-D [1] has been aimed at the development of ICRF fast wave heating and current drive systems that present a matched load to the rf transmitter despite the strongly time-varying load on the antennas. The high power transmitters typically are capable of maintaining their full rated output power only if the VSWR at the output is less than about 1.25 , while the resistive part of the antenna load impedance can vary by a factor of five or more on a submillisecond timescale in the worst case. Hence, a simple fixed impedance transformation is inadequate for this large dynamic range of load impedance. Another highly desirable property is a minimum number of variable high power impedance matching elements in the transmission line, as these bulky, complex, expensive devices are among the least reliable parts of these systems. While a system that easily satisfies these criteria can be constructed with a "combline" antenna [2], the goal of the present work is to optimize our use of the three existing four-element antennas on DIII-D. One line of development is based on the externally coupled traveling wave antenna [3]. Another method of achieving these goals is based on a simplification of the type of system presently used to power all three antenna arrays on DIII-D. In this approach, a $3 \mathrm{~dB} 90$ deg hybrid junction is used in conjunction with a two-port decoupler. The resulting system can be used for either co- or counter-current drive phasing (progressive 90 deg phasing) of the array elements, and presents a matched load to the transmitter without the use of variable tuning elements, despite variations in the resistive or self-reactive antenna loading. This approach, which will be referred to here as the balanced feed configuration (BFC), was compared with the externally coupled traveling wave configuration and with another more conventional setup in [1]. The present paper describes a practical application of the balanced feed configuration on the DIII-D tokamak, and results from the initial high power operation of this system are shown.

\section{BALANCED FEED CONFIGURATION (BFC) SETUP}

The feed circuit used to power the four-strap antenna in the 60 MHz system from 1993 through 1996 has been described previously [4], and is diagrammed in Fig. 1(a). The important properties of such a feed system can be seen from a simple model, in which the $0 \mathrm{deg}$ and $90 \mathrm{deg}$ ports (labeled 2 and 4) of an ideal $3 \mathrm{~dB} 90 \mathrm{deg}$ hybrid junction are terminated with a two-port device characterized by the $2 \times 2$ scattering matrix $S_{i j}$. We assume that the two-port is reciprocal, so $S_{12}=S_{21}$. If port 3 is terminated with an ideal dummy load, then it can be shown that the voltage reflection coefficient $\rho_{\mathrm{T}}$ seen looking into port 1 and the voltage transmission coefficient to the dummy load, $t_{13}$, are given by

$$
\rho_{\mathrm{T}}=(1 / 2)\left(S_{11}-S_{22}\right)-j S_{12} \text { and } t_{13}=-j(1 / 2)\left(S_{11}+S_{22}\right) \text {. }
$$

If the antennas, feedlines, and quarter-wave transformers are symmetric, the impedance matching networks are set identically on the two sides of the system, and the difference in electrical length between the two output ports of the hybrid

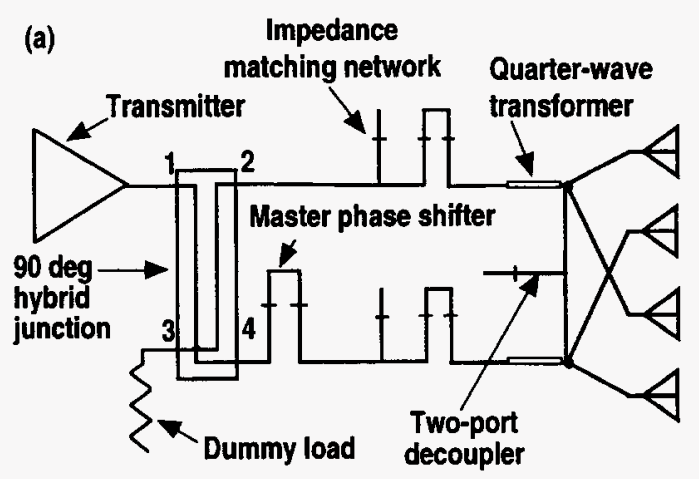

(b)

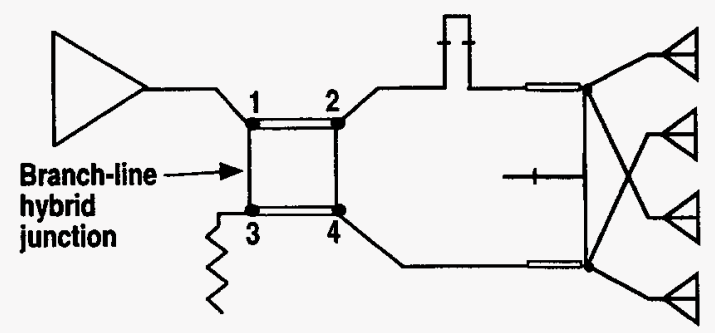

Fig. 1. (a) Transmission line arrangement used the the $2 \mathrm{MW} 60 \mathrm{MHz}$ FWCD system on DIII-D from 1993-1996. (b) Simplified arrangement tested in 1997.

\footnotetext{
*Work supported by U.S. Department of Energy under Contract Nos. DE-AC03-89ER51114 and DE-AC05-960R22464.
} 
and the matching networks is equal to an integral number of half-wavelengths, then $S_{11}=S_{22}$. The two-port decoupler can be adjusted [4] to make $S_{12}=0$. Under these conditions, (1) shows that the transmitter connected to port 1 of the hybrid sees a reflection coefficient that is identically zero, and the magnitude of the voltage transmission coefficient to the dummy load is just $\left|S_{11}\right|$. Also, the voltages at the two resonant loop feedpoints (the load side of the quarter-wave transformers) are then equal in magnitude and have a relative phasing of either $+90 \mathrm{deg}$ or $-90 \mathrm{deg}$, depending on whether the difference in length mentioned above is an even or an odd number of halfwavelengths. This produces either $[0, \pi / 2, \pi, 3 \pi / 2]$ or $[0$,$\pi / 2, \pi,-3 \pi / 2]$ toroidal phasing on the antenna array, i.e., coor counter-current drive phasing. Hence, the fundamental property of this type of system is that given the necessary degree of symmetry, the transmitter sees a reflectionless termination regardless of the magnitude of the load impedance, as long as the changes in load impedance do not break the symmetry between the two sides of the system. In practice, most of the variations in plasma load impedance on the antenna array are seen to affect each of the four elements identically. Since the decoupler is fixed during a discharge, (1) shows that variations in plasma loading that change the mutual reactance between the array elements and hence $S_{12}$ lead to nonzero reflection seen by the transmitter. Fortunately, as reported in [4], the variations in mutual from one plasma condition to another are fairly small under most conditions studied.

Since all three of the DIII-D ICRF systems were set up as indicated in Fig. 1(a) from 1994 through 1996, it proved possible to couple up to $3 \mathrm{MW}$ to an ELMing $\mathrm{H}$-mode plasma in current drive phasing by setting the impedance matching networks symmetrically [5]. However, the layout of the $60 \mathrm{MHz}$ system especially could be significantly improved for this type of operation, because the hybrid junction was located immediately adjacent to the transmitter and the two $40 \mathrm{~m}$-long transmission lines between the hybrid outputs and the quarter-wave transformers cannot be evacuated and pressurized with insulating gas for high voltage standoff, due to the type of transmission line used. Of the three DIII-D ICRF systems, only the $60 \mathrm{MHz}$ system employs Teflon insulators in the transmission lines, which means that the consequences of a transmission line arc are more serious than in the other two systems, which use quartz insulators. The large standing wave ratio that can occur transiently on the generator side of the stubs in this kind of operation can cause transmission line breakdown near any of the large number of high voltage points in the long lines, and substantial Ohmic losses in the lines due to the equally large number of current maxima in those lines. Furthermore, it was found that breakdown in the two phase shifters in the impedance matching networks was sometimes a power limiting factor. These problems could be alleviated by reducing the distance from the quarter-wave transformers to the hybrid to the minimum practical length and by eliminating the impedance matching networks entirely.

For the reasons listed above, it was decided to optimize the $60 \mathrm{MHz}$ system by relocating the hybrid junction to the other end of the two long unpressurized transmission lines and disconnecting the tuning stubs and one of the two tuning phase shifters. In this first step, the remaining phase shifter is used to switch from co-current drive to countercurrent drive phasing. Since this system operates only in a narrow band around $60 \mathrm{MHz}$, the coupled-line hybrid was replaced with a narrowband square ring hybrid junction made with the outer conductors from four standard 9" transmission line tees and four straight sections. The resulting hybrid could be mounted using standard transmission line hangers. Gas breaks located on the generator side of the hybrid permit at least moderate pressurization of the sections of line that may experience high VSWR. The resulting configuration is shown in Fig. 1(b).

In this configuration, the fraction of the transmitter power coupled to the antenna is $1-\left|S_{11}\right|^{2}$, so that the efficiency is $100 \%$ only when the reflection coefficient on the generator side of the quarter-wave transformers vanishes on both sides of the system. Without adjustable tuners, the maximum efficiency is achieved at one specific value of the resistive and reactive antenna loading. The resonant loops of transmission line with which the antennas are connected and/or the transmitter frequency must be carefully adjusted so that the impedance seen at the load side of the transformers is purely real at the specified level of reactive antenna loading, and the impedance of the transformers must be chosen so that the real part of the specified antenna load is transformed to an input impedance equal to the characteristic impedance of the remainder of the transmission line $(50 \Omega)$. The impedance of the quarter-wave sections was chosen to be $116 \Omega$, which produces a match and the maximum efficiency for an antenna loading typically measured in $\mathrm{L}$-mode discharges. The resonant loops were trimmed so that in the absence of plasma loading, the resonant frequency was $59.5 \mathrm{MHz}$; experience had shown that the typical shift in resonant frequency caused by the reactive component of the plasma loading would result in resonance near $60.0 \mathrm{MHz}$ (the frequency at which the transmitter power was optimized) with plasma. It was perhaps even more important to adjust the two loops to exactly the same resonant frequency, to maximize the symmetry of the system. By adjusting the length of a bellows section in one of the loops, the difference in resonant frequency of the two loops was reduced to about $1 \mathrm{kHz}$. At resonance, the input impedances at the loop feed points in the absence of plasma were $1380 \Omega$ and $1470 \Omega$.

The square $3 \mathrm{~dB}$ hybrid junction consists of two quarterwave long $(125 \mathrm{~cm}$ at $60 \mathrm{MHz})$ sides with characteristic 
impedance $50 \Omega$ and two quarter-wave long sides with characteristic impedance $35.36 \Omega$. Using a $5 "$ o.d. copper tube as the center conductor in a standard 9" i.d. outer conductor yields a characteristic impedance of $35.25 \Omega$. The center conductor assemblies from four standard 9 " tees were modified to accept the 5 " center conductor on the appropriate sides; the low impedance center conductors were supported only by the insulators in the $50 \Omega$ portions of the square. It proved necessary to lengthen the physical lengths of the $50 \Omega$ sides of the "square" by $18 \mathrm{~cm}$ to achieve optimum performance at $60.0 \mathrm{MHz}$, as a result of the nonideality of the tees. The measured scattering matrix elements of the final version of the hybrid agree quite well with those of an ideal $125 \mathrm{~cm}$ square hybrid. By installing the hybrid as close to the tokamak as possible, the length of transmission line between the square hybrid and the quarter-wave transformer was reduced to $8.5 \mathrm{~m}$ on the side of the system without a phase shifter, from about $50 \mathrm{~m}$ in the former configuration.

\section{HIGH POWER OPERATION OF BALANCED FEED CONFIGURATION}

After adjusting the decoupler stub and the phase shifter based on network analyzer measurements, vacuum conditioning in current drive phasing commenced. Since the quarter-wave transformers were optimized for a typical antenna loading in the presence of plasma, the magnitude of $S_{11}$ and $S_{22}$ in (1) were high with vacuum loading and most of the transmitter power in this case is dissipated in the dummy load. Specifically, the minimum value of $\left|t_{13}\right|$ is found to be 0.90 at a frequency of $59.8 \mathrm{MHz}$. The peak voltage in the system is monitored with absolutely calibrated voltage probes at the resonant loop feed points, marked "A" and "B" in Fig. 1(b). This antenna typically has been vacuum conditioned to withstand a peak $\mathrm{rf}$ voltage between 30 and $35 \mathrm{kV}$. In the BFC, we find that a transmitter output power of $1.1 \mathrm{MW}$ is sufficient to produce $30 \mathrm{kV} ; 1.5 \mathrm{MW}$ would be required for $35 \mathrm{kV}$. This power level is achievable at $59.8 \mathrm{MHz}$ without retuning the transmitter cavities from the settings that yield $2.0 \mathrm{MW}$ at $60.0 \mathrm{MHz}$.

The presence of plasma increases the resistive loading on the antennas and simultaneously decreases the inductive self-reactance of the antennas and the mutual reactance between the elements of the array. The change in mutual reactance necessitates readjustment of the decoupler stub to null out $S_{12}$ in (1). The change in required decoupler stub length from vacuum to plasma amounts to about $4 \mathrm{~cm}$, corresponding to a decrease of $33 \%$ in the mutual reactance from the vacuum value. As expected, the decrease in selfinductance caused by the plasma requires operation at $60.0 \mathrm{MHz}$ for maximum efficiency (minimum power transferred to the dummy load). An efficiency greater than 95\% with a VSWR seen by the transmitter less than 1.2 are simultaneously achieveable, as shown in Fig. 2. In this

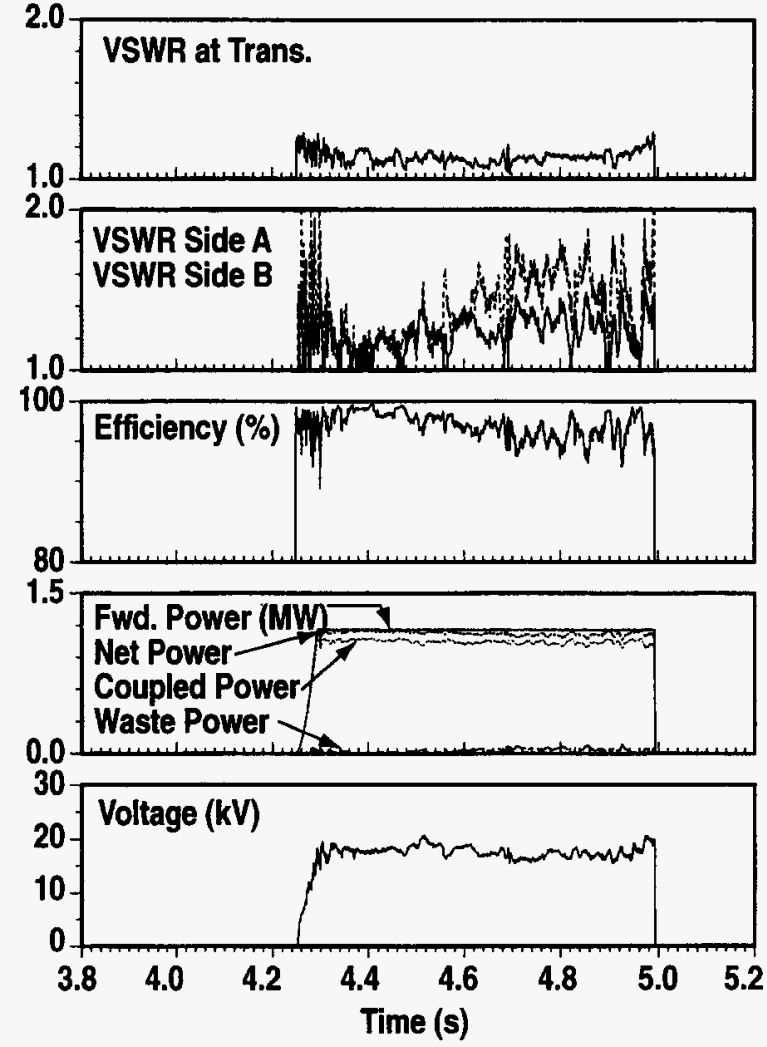

Fig. 2. Efficient operation ( $>95 \%$ of the transmitter output coupled to the antenna) of the BFC at high power into a DIII-D plasma.

example, the gap between the surface of the antenna Faraday shield and the plasma separatrix is $6 \mathrm{~cm}$. With lighter plasma loading than in this case, such as is seen with "H-mode" edge plasma conditions, the efficiency of the system is reduced, while still presenting a constant matched load to the transmitter. This is shown in Fig. 3. These data were obtained in a single plasma discharge, in which rf power level was sufficiently high to produce a transition to $\mathrm{H}$-mode confinement. The antenna loading subsequently was reduced by more than a factor of two, and the fraction of the transmitter power coupled to the antenna dropped to less than $80 \%$. However, the VSWR in the transmitter output line remained lower than 1.3 at all times, so that full power output was maintained.

Since the only two differences in the system setup from vacuum conditioning to plasma operation are a $4 \mathrm{~cm}$ motion of one stub and a slightly different frequency $(59.8$ vs. $60.0 \mathrm{MHz}$ ), vacuum conditioning during the $10-15 \mathrm{~min}$. between tokamak discharges is practical, and has been implemented. In the absence of between-shot vacuum conditioning, a gradual decrease in the antenna standoff voltage as a day of tokamak operations proceeded had been observed; routine vacuum conditioning between discharges appears to eliminate this problem; in fact, the voltage standoff improved throughout the day during one day in which this conditioning procedure was followed. 


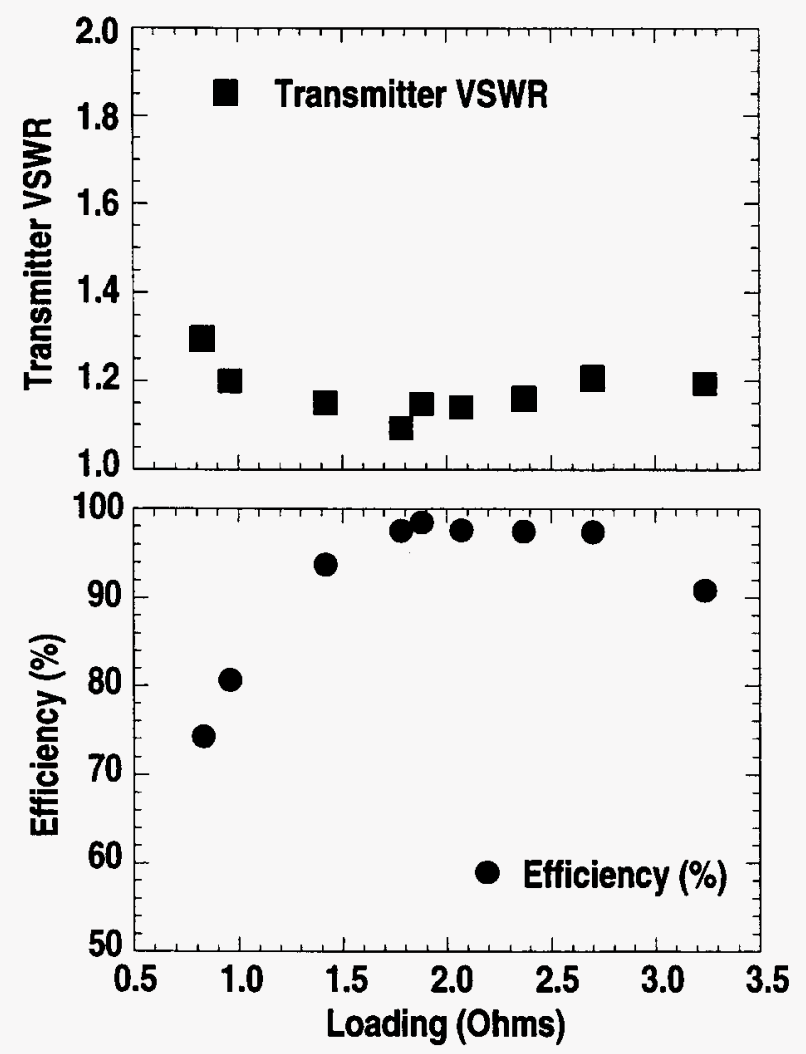

Fig. 3. VSWR in the transmission line at the transmitter output and the efficiency (percentage of the transmitter output coupled to the antenna system) as a function of the resistive antenna loading, from a single DIII-D discharge with L-mode (higher loading) and $\mathrm{H}$-mode (lower loading) edge conditions.

\section{CONCLUSIONS}

The simplified version of the DIII-D FWCD system discussed in this paper is an ideal arrangement for operating four-element antennas at 90 degree phasing with a single transmitter. This setup is particularly simple and attractive in the case of a fixed operating frequency; wideband versions require adjustable tuning elements in addition to the decoupler stub. The transmission line configuration used on the two wideband $(60-120 \mathrm{MHz})$ DIII-D FWCD systems [6] also can be operated in such a way as to retain the essential property of the BFC - the independence of the impedance match seen by the transmitter from the magnitude and phase of the antenna load impedance. In recent DIII-D experiments, all three FWCD systems have been operated successfully under conditions where the resistive antenna loading varied periodically by nearly a factor of four on a sub-millisecond time scale. A net power level of over $3 \mathrm{MW}$ was attained in this severe operating regime, with a VSWR less than 1.25 at each of the three transmitter outputs. This capability will be used in the near future to systematically study the nature of the $\mathrm{H}$-mode confinement produced by high power FWCD as the sole auxiliary heating source.

\section{REFERENCES}

[1] R.I. Pinsker, et al., "Fast wave antenna array feed circuits tolerant of time-varying loading for DIII-D," 12th Top. Conf. on Radio Frequency Power in Plasmas, Savannah, GA, 1997, to be published.

[2] R.I. Pinsker, C.P. Moeller, C.C. Petty, et al., in Fusion Technology 1996 (Proc. 19th Symp., Lisbon, 1996), (Elsevier, Amsterdam, 1997) vol. 1, p. 629.

[3] H. Ikezi and D.A. Phelps, Fusion Technology 31, 106 (1997).

[4] R.I. Pinsker, et al., in Proc. 15th IEEE/NPSS Symp. on Fusion Engineering, Hyannis, MA 1993 (IEEE, Piscataway, NJ, 1994) vol. II, p.1077.

[5] R. Prater, et al., "Fast wave heating and current drive in DIII-D discharges with negative central shear," Proc. 16th IAEA Fusion Energy Conf. (Montreal, 1996), paper IAEA/CN/64/E-1.

[6] J.S. deGrassie, R.I. Pinsker, W.P. Cary, et al., "4 MW upgrade to the DIII-D fast wave current drive system," in Proc. 15th IEEE/NPSS Symp. on Fusion Eng., (Hyannis, MA, 1993), vol. II, p. 1073. 
M98002644

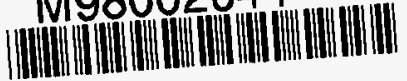

Report Number (14) $\frac{G A-A 22752}{\text { CONE-971065 - }}$

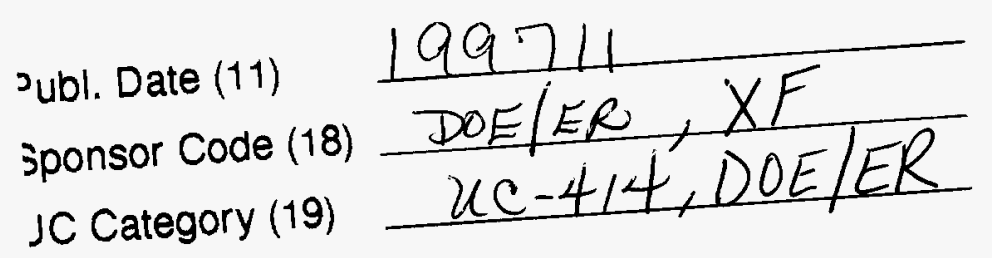

DOE 